\begin{tabular}{|c|c|}
\hline \multirow{3}{*}{ 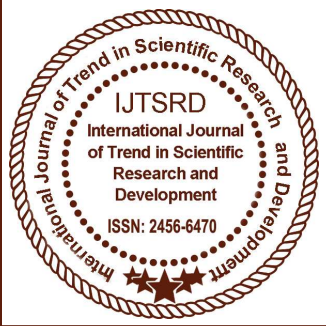 } & $\begin{array}{l}\text { International Journal of Trend in Scientific } \\
\text { Research and Development (IJTSRD) }\end{array}$ \\
\hline & International Open Access Journal \\
\hline & ISSN No: 2456 - 6470 | www.ijtsrd.com | Volume - 2 | Issue - 5 \\
\hline
\end{tabular}

\title{
Namah Subjective and Objective Aspects of Conversational Agent
}

\author{
Rajat Sharma ${ }^{1}$, Mr. Ankur Sharma ${ }^{2}$, Mr. Anurag Rana ${ }^{3}$ \\ ${ }^{1}$ Student, ${ }^{2}$ Associate Professor, ${ }^{3}$ Assistant Professor, ${ }^{1,2,3}$ Department of Computer Science \& Engineering, \\ Arni University, Kathgarh, Indora, Himachal Pradesh, India
}

\section{ABSTRACT}

Namah subjective and objective aspects of conversational agents focuses on the design and evaluation of Chatbot, a conversational agent for Natural Language Processing in An Android Application featuring a high number of different, unique characteristics: context dependent information, an support for different kind of dialogue types, i.e. chat, specific and general question answering, task oriented dialogues. The agent has currently implementations: as mobile phone application. ChatBot is an application that helps users and agents communicate easily with each other. It helps in maintaining a direct connection between users and agents. However, before this application, users get so many problems in getting their answers from the agent. The market is a communicative agent. There is no proper work done either. The user has to give their name and email address so that they can get the answer to their question and then the user gets the answer of their query after 24 hour via mail. And the information users want are either given from Google or given a link to it. so this conversational agent are easy to use. The query that the user put it will be given the correct answer to the same time or at a time. No matter how many people are logged into the questions, they will automatically get all the answers at the same time. We have set up a data set. Currently built for a one domain, we can work on this Multiplayer domain. The paper describes the modules and resources required for running the agent on both interfaces, as well as the evaluation results obtained from two assessment studies concerning the interaction design of these two agent interfaces. The feedback gathered from the studies will enable us to improve the applications in terms of service, performance and usability.

\section{INTRODUCTION}

\subsection{OVERVIEW}

Android based Conversational agent can interact with the user after understanding the necessity of user. so instead of a human on one side replying to the queries of another side the Conversational agent reply to the queries of the user. Conversational agent uses what are called neural networks, vast networks of machines that approximate the web of neurons in the human brain. It is a software application that runs automated tasks over the Internet. Traditionally chatbot performs tasks that square measure straightforward and structurally repetitive, at a far higher rate that's doable for an individual's alone. Chat larva may be a style of interactive agent, a computer virus designed to simulate associate intelligent spoken communication with one or a lot of human users via text and chat interface. The system provides precise output to its users even if minor spelling mistake is there. Moreover the parsing of sentences avoids sending to the system words that do not form a pattern. Conversational agent is essentially supported the interaction between the users and agents. UN agency moves through this humanoid application and acquire feedback instantly. This informal agent application is employed by the users over one at identical time. The AIML is build affiliation in between the humanoid and informal agent. AIML stands for computing language. Informal agent helps the users to resolve their issues simply through the assistance of informal agent application. This method conjointly give the power of correct the orthography mistakes if occur in spoken communication between the users. This method is exclusive from others with their feature of security.

A chatbot is a program that is used to participate in conversations with humans. It uses an appropriate 
interface for input and output and with the use of AI techniques it can provide realistic answers so the user will think that the communication taking place is with another human. The implementation of such systems varies from using keyword matching, string similarity or complex natural language processing techniques. More sophisticated chatbots could learn from the user input. Nowadays chat bots are used widely in web applications in order to provide help or information when it is asked by the users.

"Chatbots are computer programs that interact with users using natural languages [21]."

\subsection{EXISTING APPLICATIONS}

Chatbots are used in many establishments instead of individuals when this is almost possible.

\subsubsection{ELIZA}

The first chatbot developed was ELIZA [21]. It was established by Joseph Weizenbaum using a keyword matching method. The idea was to read the user input and search for certain keywords, if a keyword was found then the answer was retrieved. If a keyword was not present then ELIZA would try, according to specified rules, to get more information from the user to keep the conversation going. Consider the following example to understand how this would work.

\section{User: How can I get in touch with my mother?}

Then the chatbot would identify the keyword mother and would find a connection between mother and family and it would respond with a question.

\section{ELIZA: Tell me more about your family.}

The chatbot doesn't understand what it is saying. It only produces results according to rules and in this case there was a rule connecting mother and family. Then it had to produce an answer that would encourage the user to submit another question with the hope of finding a keyword. However most of the time there are some standard replies that ELIZA would use if a keyword is not found. Some examples would be "Very interesting, please go on" or "Can you think of a special example?"

The following figure gives a good example of chatting with ELIZA.

\subsubsection{A.L.I.C.E}

ALICE was realized by Richard Wallace in 1995[21]. It uses outline identical and stores the information in Synthetic Intelligence Mark-up Language, or else known as AIML, files. An AIML file is similar to an XML file that was established to store pattern acquaintance for chatbots. There are three types of AIML groups, atomic categories, default sorts and recursive categories, which are defined below.

Atomic classes: Is a type of AIML grouping where there is a particular match.

$<$ category $>$

$<$ pattern $>$ what is your name $<$ pattern $>$

$<$ template $>$ my name is Nick $</$ template $>$

$</$ category $>$

In the above example if the user submits "What is your name" then the chatbot will reply with "My name is Namah"

Default categories: Is a type of AIML category where the use of a rough character such as * is used to match any input.

$<$ category $>$

$<$ pattern $>$ what is $*<$ pattern $>$

$<$ template $>$ It is my name $</$ template $>$

$</$ category $>$

If ALICE does not find a solution using the first category it will proceed to the second. In this case if a user submits "What is a name" then ALICE will do a check until the wild char and if there is a match it will come with the answer.

Recursive categories: Is a type of an AIML category where superior tags are used to mention to a recursion to convert the user to be more detailed.

$<$ category $>$

$<$ pattern $>$ Do you know what the $*$ is $<$ pattern $>$

$<$ template $>$ It is my name $<$ srai $>$ What is

$<$ star $/><$ srai $><$ template $>$

$</$ category $>$

In this case the wild character $*$ is used to reduce the input submitted by the user. If the user submits "Do you know what the time is" then this becomes "What is the time".

The resulting figure gives an example of a conversation between a human and ALICE. 
When a user submits a question then any relevant punctuation is removed and the sentence goes through the categories to find the longest pattern match. The question "Can you tell me the time, using hours, minutes and seconds?" will become» Can you tell me the time using hours minutes and seconds" and then it will search to find the longest match. When the match is found then it will be forwarded to the output. However ALICE does not have the ability to learn and can only come with data that exist in its database.

\subsection{LEARNING CHATBOTS}

Learning chatbots have the ability to learn from user input. The aptitude of the chatbot is based on definite rules and by arrogant that the user will give a correct answer. There are two types of learning and both use the same learning rules. The first one will understand what the user said, will store it for the current session and will reply with that answer if the user asks about. The second one will store the information in its database and will reply with that answer for any user. Certain rules can be specified to understand from previous user input if the possibility of a user saying the truth is high or not and decide to store the information for future users. Also questions and answers among users can be saved in the database and compared to identify the possibility of something being true.

The following conversation gives an example of the learning procedure.

User: How many lecturers are there in the department of computer science?

Chatbot: I am sorry but I don't know the answer. Perhaps you can help me learn

User: There are 28 lecturers at the department Chatbot: Is this the correct answer?

User: Yes

Chatbot: Thank you for helping

User: How many lecturers are there in the department of computer science?

Chatbot: There are 28 lecturers at the department

\subsection{CONVERSATIONAL AGENTS}

Conversational agents are software programs those support conversational interaction between humans and machines in natural language. They help, assist, organize, manage or perform user's day-to-day tasks like booking airline/movie tickets, participating in meetings and conferences, knowing about weather conditions, getting updates about traffic in the city, checking for the best restaurants/shops around, talking to customer care service and many more. Conversational agent is a collection of behavioral components that can interpret, sense, trigger and respond or reply to user. Different terms is accustomed outline a system has the flexibility to handle user conversations like informal Agent (CA), dialog system and chat larva. CAs square measure enjoying important roles in numerous applications, for example, in selling, education, help desk, recreation, e-commerce, data retrieval and usually in business. Basically, these sorts' targeted totally different varieties of spoken languages beginning from text writing conversation, spoken language and mixed among them spoken language. As a result of this distinction, range of approaches has been projected and accustomed develop $\mathrm{CA}$ that square measure tongue e Processing (NLP), Pattern Matching (PM) and linguistics Sentence Similarity (SSS) measures. All of the $\mathrm{CA}^{\text {ee }} \mathrm{s}$ building approaches (except PM) square measure tongue dependent which implies it must perceive the targeted tongue. Understanding and computing the tongue is quit complicated and wishes totally different immense endeavor from the language students before. Chat bots, additionally referred to as informal Agents or Dialog Systems, square measure a hot topic. Microsoft is making big bets on chat bots, and then square measure firms like Face book (M), Apple (Siri), Google, We Chat, and Slack, IBM Watson Chat bots square measure too typically powerless to grasp our intentions, have bother obtaining America the right data, and square measure generally simply exasperatingly tough to upset Interacting with a machine via tongue is one in all the wants for general computing. This field of AI refers to discourse systems, spoken dialogue systems, or chat bots. The machine must give you with AN edifying answer, keep the context of the dialogue, and be vague from the human.

\subsection{CHABOT}

There are two major types of interchange systems: goal-oriented (Siri, Alexa, Cortana Watson etc.) and general conversation (Microsoft Tay bot).

Once the ontology is preprocessing is ended, it's time to imitate what kind of chatbot you would like to use. There are two main copies for a chatbot: 
$>$ Retrieval-based model - this kind of chatbot uses a fountain of predefined replies. The programmer selects suitable response based on context following a given empirical, which can be either something very simple or quite complex contingent on the condition.).

Generative model - A generative model chat box doesn't use any predefined repository. This kind of chatbot is more advanced, because it learns from scratch using a process called "Deep Learning."

\subsection{GENERATIVE MODELS}

AND

SELECTIVE

General conversation models can be simply divided into two major types:

\section{$>$ Generative models \\ $>$ Selective models}

Also, hybrid models are possible. But the shared denominator is that such models take in some judgments of discourse situation and predict the answer for this context. In the image below, you can see the design of such systems.

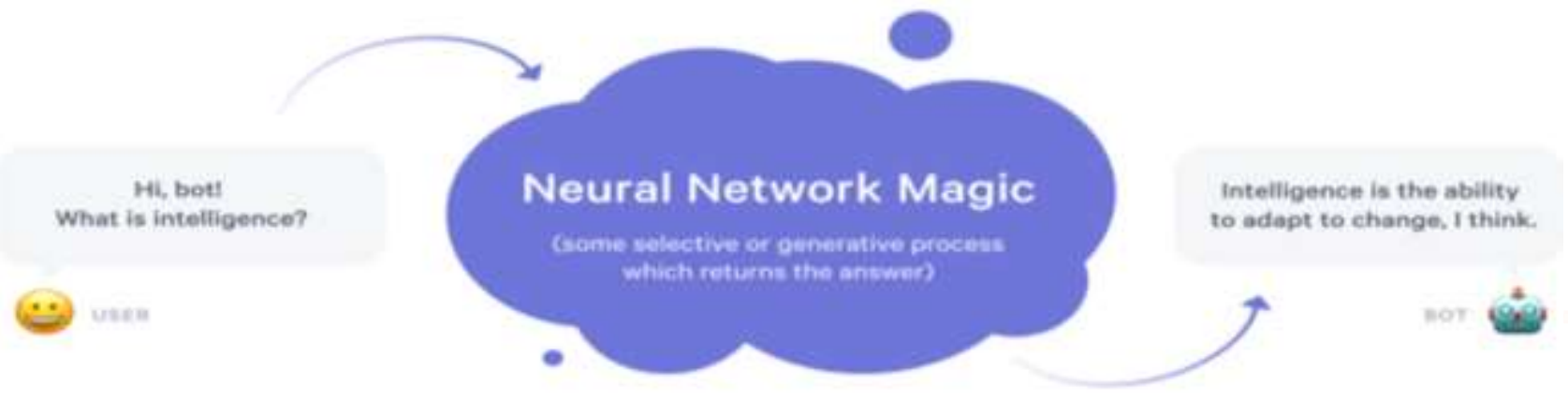

Figure -1.1 Conversation models [25]

\subsection{DIALOGUE DATA REPRESENTATION}

All models delineate below square measure trained on pairs (context, reply). Context is many sentences (or perhaps one) that precede the reply. The sentence is simply a sequence of tokens from its vocabulary.

For higher understanding, investigate the table. There's a batch of 3 samples extracted from a raw dialogue between 2 persons:

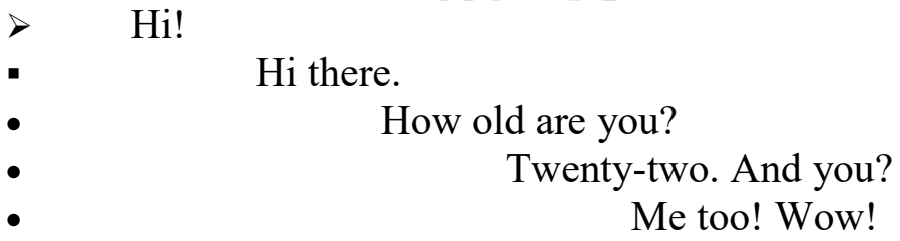

Note the (end-of-sequence) token at the top of every sentence within the batch. This special token helps neural networks perceive sentence bounds and update their internal state sagely.

Some models could use further Meta info from knowledge like speaker ID, gender, emotion, etc.

\subsection{GENERATIVE MODELS}

The informal model supported the paper A Neural informal Model. For representative discussion, this paper organized a sequence-to-sequence (seq2seq) platform that appeared at breaks the neural device adjustment field and was successfully tailored to dialogue problems. The design consists of 2 RNNs with totally different sets of parameters. The left one (corresponding to A-B-C tokens) is termed the encoder, whereas the correct one (corresponding to $\mathrm{W}-\mathrm{X}-\mathrm{Y}-\mathrm{Z}$ tokens) is named the decoder.

\subsection{MODIFICATIONS OF GENERATIVE MODELS}

A basic understanding of the sequence-to-sequence framework However will we add additional generalization power to such models? There square measure many ways:

$>$ Add more layers to the encoder or/and decoder RNNs.

$>$ Use a bi-directional encoder. There is no way to make the decoder bi-directional due to its forward generation structure.

$>$ Experiment with embeddings. You can preinitialize word set in or learn them from scratch composed with the model.

\subsection{CLASSIFICATION OF CONVERSATIONAL AGENTS}

In this section, we have a tendency to represent categories of a colloquial agent. By this classification, we have a tendency to perceive however the colloquial agent's area unit used on totally different devices and what area unit their mediums of communication with the users. We also 
looked at how they are used for different functions with totally different domains. P1 ease ask Figure a pair of. 1 that shows the classificat ion of the conversational agent.

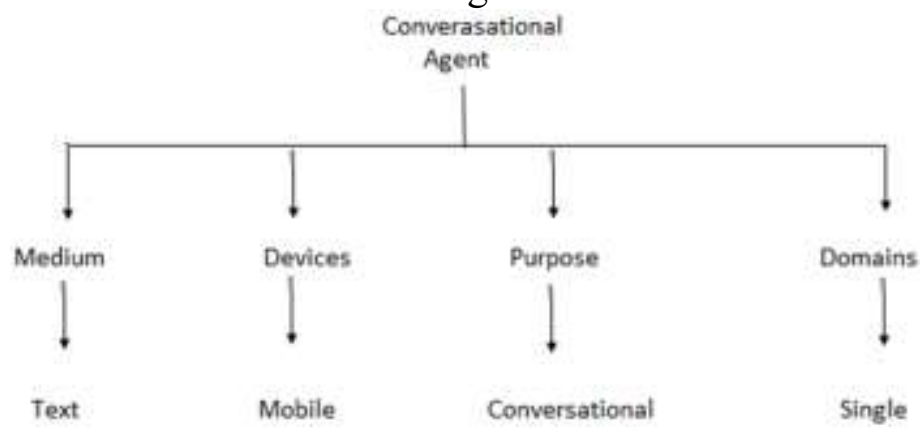

Figure 1.2: Classification of Conversational Agents

\subsubsection{MEDIUMS}

Mediums of communication are a channel of sensory input/output between a user and conversational agent. The interaction between a user and conversational agent can be text based or speech-based or it can also be based on a graphical user interface. In Text-based interaction input-output is mainly in text, in spoken dialogue systems it is speech is and in graphical user interface, interaction is based on graphical elements. The system can also be developed for multiple mediums.

\subsubsection{DEVICES}

Previously, conversational agents were used on desktops or laptops. With the increasing usage of mobile phones, they are now widely used in phonebased applications. The robot is a type of device which is talented of carrying out a difficult series factions automatically. Simulated realism devices are a truthful imitation of a three-D atmosphere that is created using communicating software and hardware. They can be knowledgeable or controlled bit hem movement of the body. This is the very effective way forth enter action with the user.

\subsubsection{PURPOSES}

Conversational agent might serve 3 purposes. First one is informational conversational agents those try to provide information to its users like travel information and timetable, weather information etc. They are used in Question-Answering systems to provide informational answers to user's questions. Second is transactional where users can perform actions with conversational agents such as purchasing a train or movie ticket, reserving a table in a restaurant etc. And third is conversational which is designed just for the sake of conversation with their users.

\subsubsection{DOMAINS}

Conversational agents are used in different domains. Based on the requirement of an application, they can be designed and developed for different domains like weather information, transport/travel information, educational domains like tutors [49], and medical domain like clinical uses for mental health [52]. We call conversational agents which serve in only one domain as single domain agents; multiple domains agent which serves in many domains, and we call them generic, if they serve for general conversations like small talk or conversation.

\subsection{CHATBOT AS A TEXT-BASED CONVERSATIONAL AGENT}

A text-based conversational agent is called a chatbot. It is using text as the only medium of communication and is used on desktops/laptops, majorly on mobile phones in different messaging applications. Messaging applications such as Face book Messenger, We Chat, Telegram, and LINE are widely used by millions of people to communicate with their friends, colleagues and companies [28]. The growing popularity of messaging platforms is driving a new era of interaction with chatbots. Messaging platforms have begun to provide functionality to develop and discover different chatbots. We currently recognize two ways of using chatbots; first are those integrated into a messaging application and second, those are using existing platforms, such as Face book Messenger, Telegram, Slack, We Chat, Viber etc. The difference is, for the second type, you can chat with the chatbots via already existing messaging application on your phone, but for the first one, you need to install and use a dedicated application to converse with a chatbot. Integrating chatbots allows these applications to communicate on the scale of one-to-one or one-to-many. Some of these messaging platforms like Telegram, Face book Messenger, Twitter are widely used as they provide their own framework for developers to develop chatbots and integrate them easily into a messaging application. We perform a short survey on four widely used messaging platforms so as to understand the methodology they provide while developing a chatbot, their features, interfaces and functionalities. We perform this survey to understand which messaging platform would be a suitable platform for implementing a chatbot in our thesis work. Table 2.2 
shows messaging platforms and their different attributes.

\section{REVIEW OF LITERATURE}

R. Mahapatra et al. [1] describe the system is utilized the power of chat bots to serve interactive internet in enterprise applications. It's showcased a reference model to utilize GIS in E Governance system and embody core Land Record Maintenance and Extension module. It alternative ASE (Artificial Support Entity) module as associate External Extension that communicates with core IEGS (Integrated E-Government System) victimization SOA protocols. The chat bot consists of a set of AIML files which will be a stimulusresponse model. It's supported Program E and uses PHP as facet stores AIML patterns in MYSQL data and mythical being uses VI application. It consists of animal Vser Interface, associate interface and AIML data. It put together tries to mimic real human conversations victimization Persona-AIML style that consists of categories Base, temperament half, Dialogue Log, and Reasoning half.

Shawar BA, et al. [2] describe that computing lan guage (AIML) springs from protractile language (XM L) thatisemployed tocreate up colloquial agent(Chabo t) unnaturally. There ar developed heaps ofworks tofor m colloquial agent. However low value, configuratio $\mathrm{n}$ and availableness alter to use it in numerous applications. During this paper, we tend to provide a temporary review of some applications that are used AIML Chabot for his or her colloquial serviceSo, th is can be well liked day by day with enterpriseran d users to produce economical service.

Augello et al [3] Core of the system may be an informal agent that is in a position to speak to tourer in natural languages. It's supported 2 main parts: chatbot module and multimodal boundary. First, chatbot instrumentality is obligatory through Alice Technology and it's connected with Open Cyc Technology for sense philosophy content. It's been enforced a brand new terminology known as XHTML+Voice. Automatic Speech Recognition (ASR) and Text-to-Speech (TIS) technologies square measure embedded into multimodal browser. Speech method is applied through associate ad-hoc inbuilt synchronic linguistics in line with W3C Speech Recognition synchronic linguistics specification. Second sub module is RFID module that is calculable electronic device position at intervals the setting. To carry knowledge into a tool, there square measure employing a device known as RFID tags. RFID tags categorizes into active and passive. This is often used passive RFID tags that operate while not external power resource and every having distinctive ID.

D. De Orlando et al [4] it's associate integrated platform include with basic AIML information, PABX (Private machine-controlled Branch exchange) for VoIP phone management and Servers management daemon. During this case, chatbot is named Tutorbot as a result of its practicality backing of didactics tired e-Iearning environments. It depends its main options on an outline of data in AIML and contains some options like tongue management, presentation of contents, and interaction with computer program. Besides, the complete e-Iearning platforms' operating is joined to indispensable services above all to internet service. It's been created continuous watching service on e- Iearning platform servers that is another dominant machine known as Daemon. If there square measure any anomalies on the server that contain e- Iearning platform, a requirement of emergency decision to a quick intervention of technical support that manages PABX. The computer code of PABX is chosen by Asterisk that permits the management regards some essential practices to our aim.

F. Mikic et al [5] Intelligent instructional system \&\#40;INES\&\#41; may be a practical epitome of an internet learning platform, which mixes 3 essential capabilities, Learning Management System \&\#40;LMS\&\#41;, Learning Content Management System \&\#40;LCMS\&\#41;Intelligent Tutoring System \&\#40;ITS\&\#41;. It consists of various tools and technologies. CHARLIE (Chatter Learning Interface Entity) is AIML based mostly intelligent chatterbot and supported program D and uses Ajax technology to take care of Asynchronous communication that permits interactive application or RIA. Intelligent agent supported BDI (Believes, Desires, and Intentions) that acts as brain of the system. Besides, associate abstract thought engine supported JESS (a rule engine supported Java platform) and decides what's allowed to try and do. At last, metaphysics may be a part of INES that semantically defines contents of the courses. Linguistics managing users, contents tools also are utilized in this technique. 
S. du Preez et al [6] This Web-based system is employed intelligent voice recognition capabilities and recording machine approaches. It controls communication structure with internet service and consists of 3 components: consumer, server, content acquisition. The server may be an easy object access protocol (SOAP) aware web application that isolates consumer from interacting inner workings. All messages square measure formatted in associate XML and encapsulated in SOAP text based mostly message pack. The consumer contains voice recognition process module. This application will simply accessible by consumer browser and user is allowed to register and login to the system. Once the user logs the system, the system can greet the user by default and prepare to receive question or statements. ALICE larva engine is employed to meet this functions that contain a collection of AIML files. To help the coaching, coaching modules, Third party knowledgeable system, final analysis Assistant was used however to refine question self-processing AIML-assisted classes square measure used. Besides, there square measure enforced consumer application to produce higher service. It consists of 2 options: text-input, voice-input. It conjointly needs variety of libraries for process voice inputs and secured communication with internet service. In application management method, there square measure many parts that square measure to blame for consumer interruption. Management unit of request will start to occasion to activate either line or voice credit module or SOAP statement module. So, the customer sends and receives plain text. Internet service processes received queries all response generation modules.

\section{Augello et al [7] Framework for Agent-based} Semantic-aware Interoperability (FRASI) is streamlining of the chatbot mental object and resulting in sentence analysis method. This questionanswering system uses pattern matching chatbot technology. It consists of atomic, default, metaphysics based mostly, and supreme default. However this contains some limitations. to scale back limitations, it projected 3 modules to use with chatbot. Increased Symbolic reduction (ESRAI) preprocesses sentences and reduced them into less complicated structure. A second module, named bootstrapping module will exploits ontologies so as to mechanically extend Chatbot K. Third module is CYD module that makes it doable to use ontologies to form dynamically answers generated by abstract thought process of user question.
S. Negi, S. Joshi et al [8] this technique is works as task-oriented tongue dialog system that is act with user to accomplish a given objective. During this case, there's projected unattended, apriority like algorithmic program that extracts subtasks and their valid orderings. so as to extract subtask from a corpus, this model is accountable to try and do this. First, it's required to cluster and canonic Alize similar vocalization semantically to interchange all the name entity by their sort and generate a feature vector for every vocalization. Feature vectors square measure clustered to cluster along semantically with similar vocalization. This method is named vocalization standardization. Second, there square measure discovered subtask kind standardization calls then finding precondition (utterance pre condition, flow pre condition) for every subtask. This method is decisioned|is named is termed $\}$ mining subtask from call transcripts. Third, Extracted task structure is encoded as associate AIML construct to create taskoriented chatbot. This method is named AIML Generation.

J. T. C. Tan et al [9] this framework relies on simulation platform named SIGVerse. It's divided into Server System, consumer System, Service suppliers. In Server System, SIGServer is that the center of simulations and it runs core application and agent controller is works as agent intelligence. Then, in service suppliers, it offers additional choices. During this case, Dialogue engine and behavior recognition module square measure developed and face expression, speech feeling is beneath development. Besides, Dialogue Engine utilizes AIML and behavior recognition module is employed Mimesis model with Hidden Andre Mark off Model.

Augello et al [10] humorists larva is informal agent that is provided sense of humor by telling mirthful account to user and capable to listening jokes, attempting to know mirthful level. It's a collection of ordinary Alice classes which permit holding a general oral communication with user. It's conjointly another set of classes that aimed toward humorist sentence generation. Next, it contains a collection of Chatbot to acknowledge mirthful intent with external resources and CMU pronunciation lexicon. This agent is especially tried to mirthful act with user.

M. D. Leonhardt et al: [11] Dorothy chatbot isn't solely interacts with user however conjointly manage network via management protocols. It's used 
supported ALICE resolution. ALICE integrated with Dorothy by generating five parameters that square measure topic, address, operation, time and scenario. This technique consists of 3 modules: central module, PBO module and network history info module. Central module is accountable to receive all info generated by ALICE. Network history info module consists of collect module, history info base. Collect module collect info of network and history info information. History info base is employed to gather measure of network between intervals. PBO module relies on case-based reasoning and downside resolution.

G. Pilato et al [12] this is often combined Latent linguistics Analysis with sense and cognitive content illustration to boost dialogue capabilities. During this case, agent space is including 2 spaces: rational area, associative space. Rational space composed of AIML $\mathrm{K}$ and CYC metaphysics. On the opposite hand, associative space is obtained with mapping CYC construct as vectors into linguistics area engineered by means that of Latent linguistics Analysis. Given a selected $\}$ small theory this is often assortment of ideas and facts of particular domain and linguistics area inferred from corpus text. Corpus is made each unexpected extracted pages from Wikipedia repository with specific CYC small theory.

Every construct projected on the realm and reciprocal geometric distance between ideas defines subsymbolic relationship internet that's seen as sub symbolic linguistics layer automatically alternative CYC philosophy. So, chatbot can act with user exploiting its customary and CYC metaphysics however it will use of its assertive reasoning space to retrieve linguistics ideas between metaphysics ideas that square measure simply approachable through associative sub-symbolic ways.

G. Pilato et al [13] this informal agent relies on standard information illustration and proof-of-concept epitome. Its hybrid model and include completely different information illustration and reasoning capabilities that composed of various components: dialogue engine, dialogue instrument, and tract. The goal of dialogue engine to separate ancient monolithic mental object into completely different parts known as modules. Every module of the dialogue engine has its own abstract thought engine and mental object composed of Alice technology. Besides, the framework is general purpose. Dialogue instrument is capable to capture specific feature concerning context of dialogue and manage them as variables. There square measure principally four sorts of context variables: topic, speech act, user, and goal. Tract is employed as module selector and a planner. Standard selector will change or disable dialogue module to pick a lot of acceptable module. Besides, a planner uses a selected context variable so as to outline temporal evolution of every module.

Sarthak et al. [14] describe the A chatbot is a conversational agent where a computer program is designed to simulate an intelligent conversation. It will take user input in several formats like text, voice, sentiments, etc.

For this purpose, several open supply platforms area unit obtainable

Computer science terminology (AIML) comes from $p$ rotractibleterminology (XML) that is employed to $\mathrm{cr}$ eate up a informal agent (chatbot) unnaturally. durin gthispaper, we tend to use program that is associate degree AIML interpreter for the generation of the responses of users input. we've used this technique for developing associate degree mechanical man application chatbot which can act with user mistreatment text and voice responses.

Shawar.BA,xetxalx [15]xDescribexthat computerscie nce terminology (AIML) comes from protractibleterm inology (XML) that isxemployed toxcreate up inform al agent(chatbot) unnaturally.

There area unit developed loads of works to form informal agent. However low price, configuretion and convenience modify to use it in varied applications. during this paper, we tend to provides a transient review of some applications that area unit used AIML Chabot for his or her informal service.

Bayan Abu Shawar et al. [16] describe that a chatbot is a conventional agent that is able to interact with users in a given subject by using natural language. The conversations in most chatbot are still using a keyboard as the input. Keyboard input is considered ineffective as the Conversation is not natural without any saying and a conversation is not just about words. Therefore, this paper proposes a design of a chatbot with avatar and voice interaction to make a conversation more alive. This proposed approach 
method will come from using several API and using its output as another input to next API. It would take speech recognition to take input from user, and then proceed it to chatbot API to receive the chatbot reply in a text form. The reply will be processed to text-tospeech recognition and created a spoken, audio version of the reply.

Wei Liu et al. [17] describe that nowadays, more and more chatbots can get acquainted with the world by equipping with knowledge rich minds which connect to Internet. In this paper, we will first explore how to integrate more knowledge into chatbot and how to make chatbot be aware of what are going on in the world. On top of that, a series of evaluations were performed on the prototype system. Through the evaluations, we mainly want to validate the proposed method in the prototype system and how the abundant level of knowledge accumulation of a chatbot affects the interactions between the chatbot and the users from the user satisfaction aspect. Experiment shows that the proposed method not only broadens the knowledge boundary of chatbot, but also makes the talks between users and chatbot more coherent. In addition, it shows that the level of user satisfaction is proportional to the amount of fact knowledge.

Supratip Ghose et al. [18] describe that in this work, we explain the design of a chat robot that is specifically tailored for providing FAQ Bot system for university students. In this paper, we model the Information Repository by a connected graph where the nodes contain information and links interrelates the information nodes. The design semantics includes AIML (Artificial Intelligence Markup Language) specification language for authoring the information repository such that chat robot design separates the information repository from the natural language interface component. Correspondingly, in the research, we built three untried organizations Consequently, the information repository can easily be modified and focused on particular topic without recreating the code design. New parameters and consequence recommends that topic exact dialogue attached with informal knowledge yield the all-out dialogue session than the overall conversational dialogue.

John P. McIntire et al. [19]describe that Distributed text-based communications (e.g., chat, instantmessaging) are facing the growing problem of malicious "chatbots" or "chatbots" (automated communication programs posing as humans) attempting social engineering, gathering intelligence, mounting phishing attacks, spreading malware and spam, and threatening the usability and security of collaborative communication platforms. We provide supporting evidence for the suggestion that gross communication and behavioural patterns (e.g., message size, inter-message delays) can be used to passively distinguish between humans and chatbots. Further, we discuss several potential interrogation strategies for users and chat room administrators who may need to actively distinguish between a human and a chatbot, quickly and reliably, during distributed communication sessions. Interestingly, these issues are in many ways analogous to the identification problem faced by interrogators in a Turing Test, and the proposed methods and strategies might find application to and inspiration from this topic as well.

Ly Pichponreay et al. [20] describe that with rapid development of information and communication technology, people are very diverse in education, learning style, and knowledge improvement methods. This paper presents an approach of converting documents into knowledge of Chatbot system that enables users to make more benefits of it by asking and answering questions through the use of electronic documents integrated with simulate system. It is an integrated system for enrich contents of documents from popular format such as Portable Document Format (PDF) and digital photos. The workflow of this system is started from extracts texts using Optical Character Recognition (OCR) from files, and then generates questions via over generating Transformations and Ranking algorithm, and finally let Chat-bot response to the user's question when it is matched with the String pattern.

Galvão et al. [21] proposed the Persona-AIML architecture for developing Chatbots using AIML, with a personality. Personality elements such as attitude, emotion, mood, physical traits and are incorporated into the Chatbot. The response to the user is greatly influenced by these traits. The main advantage of this architecture is flexibility in creating Chatbots with different personality. The efficiency of the bot was verified by the users of the Chatbot in Internet Relay Chat (IRC) environments and the web.

Datta et al. [22] proposed yet another Chatbot model, which combines the features of A.L.I.C.E., Quartet architecture and the internet. The proposed Chatbot 
acts as an information retrieval system and has the advantage of being a generic Chatbot that can efficiently converse in natural language, by using the Bayesian Network Model, for smoothly navigating amongst different conversation topics. It makes use of the internet as its data corpora.

Lokman et al. [23] have proposed a Virtual Dietitian Chatbot (ViDi) for diabetic patients. ViDi makes use of Question and Answer pattern matching system. In order to provide correct diets, ViDi is made to keep track of the user's conversation history through the use of a parameter called as vpath, which tells the path the user takes, during the QA session, across different levels. This gives ViDi the power to respond based on the entire conversation.

Kethuneni et al. [24] The Chatbot is accompanied by a Nonverbal Behaviour Generator (NVBG) avatar, which induces a personality to the bot. The primary function of the AIML based Chatbot is to provide health guidance by retrieving data from open source health information systems.

Rahman et al. [25] uses a tailored version of A.L.I.C.E. as a domain specific FAQ bot for developing student information system. The knowledge base consists of new AIML files of different categories apart from A.L.I.C.E.'s base knowledge, with the provision of navigating from one category to another. The bot is also capable of remembering the conversation.

Rosmalen et al. [26] showed that it is possible to create and integrate Chatbots with other environments, in this case, EMERGO - a serious gaming environment that is used to offer case studies to train students. The Chatbot acts as an interface between the user and EMERGO and provides resources from EMERGO based on the user's request.

Khanna et al. [27] assess the strengths and weaknesses of current AI systems through the use of Chatbots. Apart from the regular functions provided by Chatbots, the authors have added additional functionality such as embedding applications such as a calculator, data file handling and error handling to make the bot an efficient. The drastic limitations to the current AI systems are discussed along with counter measures that need to be inculcated to redefine AI.
Yu et al. [28], is a multimodal dialog system which was designed to keep users engaged in a social conversations as long as possible. The system uses confidence scores to guide the direction of the conversation. The system is supported by various other technologies such as Google Automatic Speech Recognition, Multi Sense etc., to collect auditory and visual data from the user. These data are used to evaluate the user's engagement. Hand-coding of vast knowledge to create an efficient Chatbot is a cumbersome activity. To overcome this limitation.

Shawar et al. [29] developed a program to convert different types of corpora, such as dialogue, monologue and FAQ, into AIML categories. The result is an extended range of knowledge base for the Chatbot which gives it the flexibility to operate in different domains and serve different purpose.

In Hatwar et al. [30] have proposed a Chatbot that will serve as a shopping guide. The proposed Chatbot, which works on the web, will provide various details such as on-going sale and also provides navigation features to the desired shop.

Behera et al. [31] Talks about Chappie, a semiautomatic intelligent Chatbot. The author classifies the Chatbot as intelligent because it satisfies the three basic criteria for an intelligent system, namely: Understanding the intent of the user; ability to handle repetitive questions efficiently; and routing the conversation to a human agent. The resulting Chatbot had a positive effect and was able to fool people into thinking that it was a human.

Mhatre et al. [32] were able to create a web based Chatbot, Donna, which acts as a personal assistant and helps users in setting up meetings. Unlike few Chatbots which depend on e-mails being forwarded to it in order to process them, Donna automatically takes care of these functionalities. Donna gets the work done with help from Gmail API and Google Calendar API respectively. Donna proved to be an effective assistant.

V. Doshi et al. [33] present the model of an Android application Chatbot. The Chatbot uses AIML and Android application with a JSON parser acting as the middleware API. The resulting Chatbot has the advantages of both AIML and Android. This research has shown that Chatbots could be developed using a single platform and can be used in other platforms 
provided there is sufficient middleware. This takes us one step closer towards designing platform independent Chatbots.

\section{RESEARCH WORK}

\subsection{PROBLEM FORMULATION}

Conversational agent is basically based on the interaction between the users and agents. I have researched and analyzed this project to my best knowledge. And I came to know that all the chatbots in the current market do not give the correct answers to the user's queries. Who interact through this android application and get feedback instantly. However, this android application is also help in finding the location of users. On the other hand, in this android application there is user on the one side and the conversational agent on another side. This conversational agent application is used by the users more than one at the same time. The AIML is make connection in between the android and conversational agent. AIML stands for artificial intelligence mark-up language. Conversational agent helps the users to solve their problems easily through the help of conversational agent application. This system also provide the facility of correct the spelling mistakes if occur in conversation between the users. This system is unique from others with their feature of security. Moreover, before my application users face so many problems in communication directly to the opponents. Such as, users send their query to the agent but agent never gave the immediate response. They took some time then gave the answers of the users queries. However, with the chatbot application users can directly communicate with the agents and can get the immediate response from the agents. It saves a lot of time of both the parties. This application software helps in solving the problems of the users with immediate answers from the agents.

\subsection{RESEARCH WORK}

Chat bots are searched for the convenience of users and customers, who are known as the objectives of Namah Topic and Conversation Agent. These Chat Bots users can get answers directly from agents without any delay and inconvenience. However, before this Chatbot users get so many problems in getting answers to their questions because the agent did not respond immediately. Agent takes so much time to respond to customers but now, the talkative agent's subjective and objective aspect provides direct answer facility and save users time. In this research and analysis for my best knowledge in this project, it has been stated in this research that not all Chatbots in the current market do not answer the user's answers correctly. To overcome this, worked in this project to improve it so that the user can easily use it with complete satisfaction. It created a data set for it and trained the chatbot in such a way that it would answer the questions of all users accurately. The conversation agent is basically based on the interaction between users and agents. First of all, in developing this application by developing the conversation agent "chatbot" in Android studio. This negotiating agent application works easy. Then with the help of artificial intelligence, the data sets were used in this project in which we put all the questions. With the help of user queries, this chat agent will answer the application exact. Users in this application will ask their questions through chat option. This project connects all the answers to those questions through the Data Set in the application. Then match the questions with the answers and the exact result will be provided by the users. This app will make the user experience easier and they will easily get their answer and feedback. There is no chatbot in today's market that can answer the user's exact answers. That's why we will train this chatbot in such a way that it will first be able to read and understand the user's query and then give an accurate answer. It does not matter which language users are asking for a query, it will respond in the same language in which users are asking for queries. We are currently working on a single domain. But later it will be available to work in hotels, hospitals etc. The conversation agent is basically based on the interaction between users and agents. Who interacts with this Android application and receives immediate feedback. This conversation agent has used more than one user at the same time. AIML is making connections between Android and Conversation Agent. AIM stands for artificial intelligent markup language. The conversation agent helps users solve their problems easily with the help of a chat agent. This system also provides the ability to correct spelling mistakes when in conversation between users. This system is unique with the convenience of others. The chatbot is the application which helps the users and agents to communicate with each other in easy manner. It helps in direct relation between users and agents. However, before this application users face their responses from agent. Now, I develop a chatbot application to provide users with comfort. Users can send questions directly to the agent and get an immediate response from the agent. I developed this app in Android studio for 
questions. Users can send their questions to chat options and the agent can easily send feedback so this question and answer are converted into conversation. For these questions I used the data set in which I kept all the questions. This chat agent application will answer the exact answers with the help of user queries. These days, there is not so much chatbot in the market. So, my ChatBot application will give users easy experience and easy feedback. A chatbot is a conversation agent where a computer program is designed to emulate an intelligent conversation. It can take user input in many formats like text, voice, emotions etc. For this purpose, many open-source platforms are available. The market is a communicative agent. There is no proper work done either. The user has to give his /her name and email address so that he/she can get the answer to his/her question. The user has to give their name and email address so that they can get the answer to their question and then the user gets the answer of their query after 24 hour. And the information users want are either given from Google or given a link to it. so this conversational agent are easy to use. The query that the user put it will be given the correct answer to the same time or at a time. No matter how many people are logged into the questions, they will automatically get all the answers at the same time. We have set up a data set. Currently built for a one domain, we can work on this Multiplayer domain.

\subsection{OBJECTIVES OF STUDY}

The objective of study is listed as under:

$>$ User can understand easily to the chatbot application and easily they can use this application.

$>$ User can ask query in normal chatting language (English).

$>$ User can ask query Without Internet (Offline Data set).

$>$ It's Totally Free of Cost and Easy To use or User Understanding.

In this researched and analysed this project to my best knowledge In this researched that all the chatbots in the current market do not give the correct answers to the user's queries. To overcome this thing, In This project worked on make it better so that user can use it easily with full satisfaction. In this made a data set for this and trained the Chatbot in such a way that it will give accurate answer to all the users' queries. Conversational agent is basically based on the interaction between the users and agents. Firstly In
This Project to develop this application by developing the conversation agent "Chatbot" in Android Studio. This conversational agent application work easy. Then with the help of artificial intelligence In this project which used the data sets in which We kept all the queries. With the help of user queries, this conversational agent application will give the accurate answer. In this application user will ask their queries through chat option. This project which add all the answer those queries through data sets in the application. Then match the queries with the answer and accurate result will be providing by users. This application will make the user experience easy and they will get their answer and response easily. In today's market there is no such Chatbot that can give the accurate answer to the user's queries. So we will train this chatbot in such a way that it will first read and understand user's query and then give the accurate reply. No matter in which language users are asking query, it will respond in the same languages in which user are asking the query. Currently we are working on single domain. But later it will be available to work in hotels, hospitals, etc.

\subsection{METHODOLOGY}

Text input is processed by a software function called a "classifier", this classification associates an input sentence with an "intent" (a conversational intent) which produces a response.

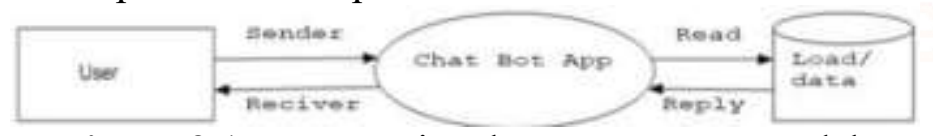

Figure.3.1 Conversational Agent Process model

Think of a classified as the simplest way of categorizing a bit of knowledge (a sentence) into one amongst many classes intent). The input "how square measure you?" is assessed as associate intent that is related to a response like "I'm good" or (better) I am well We learned regarding category fiction early in elementary science: a pong id is within the class "mammals", a New World jay is within the category "birds", the world is within the category "planets" and then on. Generally speaking, there square measure three totally different forms of text classifiers. Think about these as computer code machinery, engineered for a particular purpose, just like the drum of a musical box. The pro for victimization retrieval-based model is that the incontrovertible fact that it won't build grammatical mistakes, however, it'll be rigid too, and so not going to seem ,human”. Generative models, however, don't guarantee either to seem ,human”, but they'll adapt higher to stunning demands and queries 
from customers. Gratuitous to mention, a generative model is more durable to good, and our current state of information will get U.S.A. solely to this point. For now, going with a generative model choice means that accepted that you simply won't be able to build an ideal chat larva for each task.

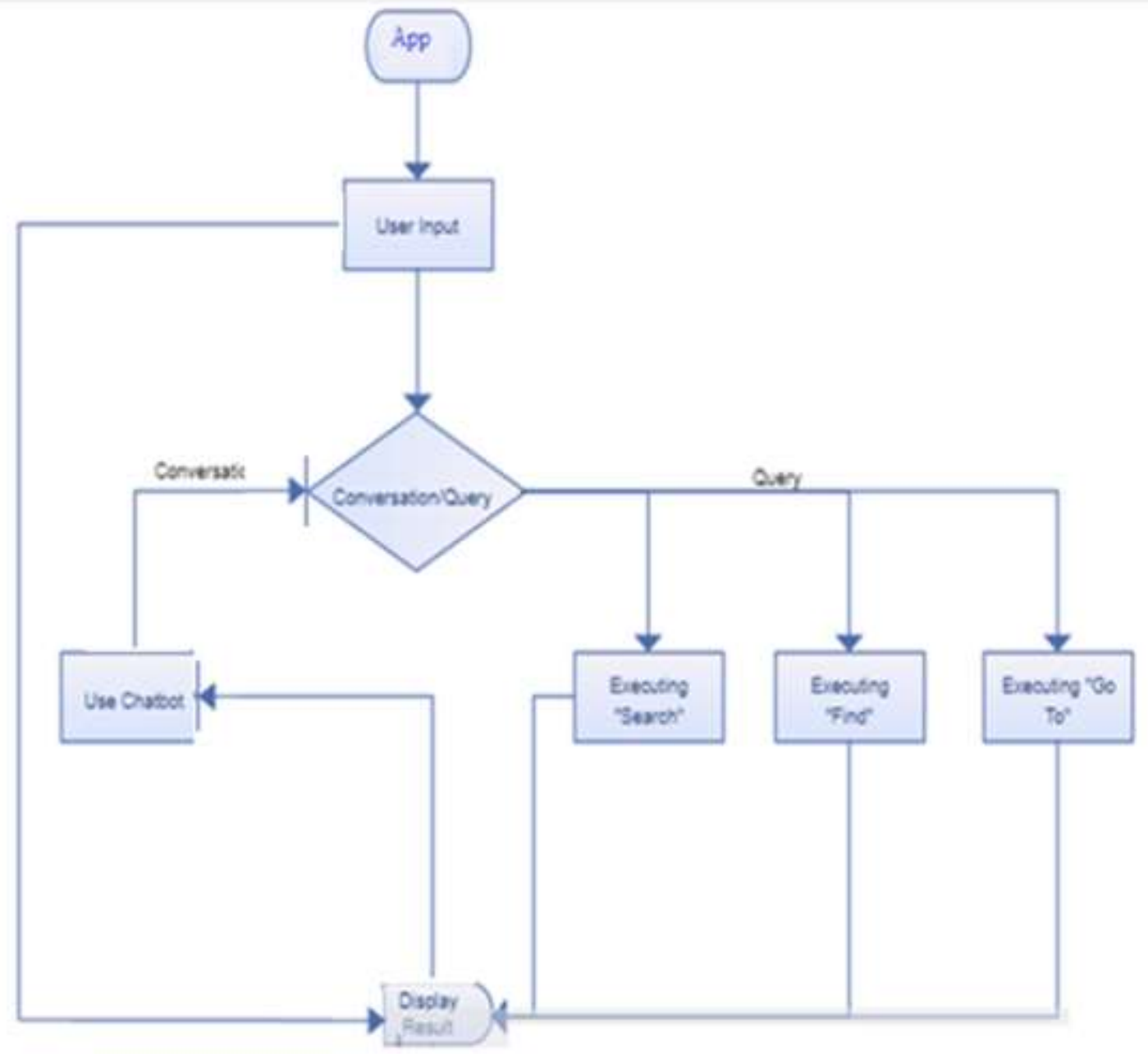

Figure.3.2 Conversational Agent Architecture

\subsection{TRAINING DATA}

When we develop a chatbot for a client we tend to train the Chat bot in five stages:

1. WARM-UP TRAINING -this is often wherever we tend to use shopper knowledge to induce the chatbot as sensible as we will to induce started (and this is often wherever most firms either stop or do not even start!).

2. REAL-TIME TRAINING - this is often wherever we tend to analyze incoming conversations in time period. It tells North American nation what folk area unit is saying/asking the larva, instead of what we predict they'll say/ask. This is often wherever we tend to use shopper knowledge to induce the chatbot as sensible as we will to induce started (and this is often wherever most firms either stop or do not even start!).

3. SENTIMENT TRAINING -this is often wherever we tend to train language and functions supported however folks area unit rebuke the chatbot. As a basic example, if we tend to find a cross user we tend to use totally different responses to if we tend to find a contented user.

4. EFFECTIVENESS TRAINING -this is often wherever we glance at the results of conversations to coach a way to get folks thereto result. Sounds cryptic i do know, ultimately, it means that we tend to learn what a decent result of a spoken language is and alter language, options and responses to induce a lot of folks thereto result quicker.

5. CONTINUOUS IMPROVEMENT - this is often wherever we tend to do all of the previous coaching in a very electrical circuit. We tend to unceasingly learn from user interactions, results and feedback and improve a chatbot's language, spoken language flows and functions. The a lot of complicated a chatbot, the foremost investment there's in iteration and continuous improvement. 


\subsection{DEEP LEARNING APPROACH}

Chatbots that use deep learning area unit the majority victimization some variant of a sequence to sequence (Seq2Seq) model. In 2014, Ilya Sutskever, Oriol Vinyals, and Quoc lupus printed the seminal add this field with a paper called "Sequence to Sequence Learning with Neural Networks". This paper showed nice ends up in AI specifically, however Seq2Seq models have big to comprehend a spread of IP tasks. A sequence to sequence model consists of two main elements; associate degree encoder RNN and a decoder RNN (If you're a bit shaky on RNNs, look at my previous diary post for a refresher). From a high level, the encoder's job is to encapsulate the data of the input text into a set illustration. The decoder's is to require that illustration, and generate a variable length text that best responds to that. As you keep in mind, associate degree RNN contains variety of hidden state vectors, that every represent info from the previous time steps. For instance, the hidden state vectors at the third time step are going to be performed of the primary 3 words. By this logic, the ultimate hidden state vector of the encoder RNN may be thought of as a reasonably correct illustration of the entire input text. The cell's job is to require within the vector illustration $\mathrm{v}$, and choose that word in its vocabulary is that the most applicable for the output response. Mathematically speaking, this implies that we tend to reckon chances for every of the words within the vocabulary, and select the argmax of the values. The second cell are going to be performed of each the vector illustration $\mathrm{v}$, additionally because the output of the previous cell. The goal of the LSTM is to estimate the subsequent contingent probability. The left aspect refers to the chance of the output sequence, conditioned on the given input sequence. The correct aspect contains the term $\mathrm{p}(\mathrm{yt} \mid \mathrm{v}, \mathrm{y} 1 \ldots \mathrm{yt}-1)$, that could be a vector of chances of all the words, conditioned on the vector illustration and therefore the outputs at the previous time steps. The Pi notation is solely the multiplication equivalent of letter (or summation). The correct hand aspect may be reduced top (y1|v) * $\mathrm{p}(\mathrm{y} 2 \mid \mathrm{v}, \mathrm{y} 1) * \mathrm{p}(\mathrm{y} 3 \mid \mathrm{v}, \mathrm{y} 1, \mathrm{y} 2)$. Let's check a fast example before moving on. Let's take the input text we tend to saw within the 1st image. Given the phrase "Are you free tomorrow?" let's have faith in however the majority would answer the question. A majority can begin with one thing on the lines of "Yes", "Yeah", "No", etc. once we're done coaching our network, the chance $\mathrm{p}(\mathrm{y} 1 \mid \mathrm{v})$ are going to be a distribution that appears just like the following. The second chance we'd like to reckon, $\mathrm{p}(\mathrm{y} 2 \mid \mathrm{v}, \mathrm{y} 1)$, are going to be a perform of the word this distribution y1 as well because the vector illustration $\mathrm{v}$. The results of the Pi (product) operation can offer North American nation the foremost doubtless sequence of words that we'll use as our final response. One of the foremost necessary characteristics of sequence to sequence models is that the skilfulness that it provides. Once you think about ancient millilitre strategies (linear regression, SVMs) and deep learning strategies like CNNs, these models need a set size input, and manufacture mounted size outputs additionally. The lengths of your inputs should be identified beforehand. This is often a big limitation to tasks like AI, speech recognition, and question responsive. These area unit tasks wherever we do not understand the scale of the input phrase, and we'd conjointly wish to be ready to generate variable length responses, not simply are affected to at least one specific output illustration. Seq2Seq models give that flexibility.

\subsection{DATASET SELECTION}

When pondering applying machine learning to any form of task, one among the primary things we'd like to try to is contemplate the kind of dataset that we might have to be compelled to train the model. For sequence to sequence models, we'd like an outsized range of oral communication logs. From a high level, this encoder decoder network must be ready to perceive the kind of responses (decoder outputs) that square measure expected for each question (encoder inputs). Some common datasets square measure the Cornell pic Dialog Corpus, the Ubuntu corpus, and Microsoft's Social Media oral communication Corpus.

\subsection{DATASET CREATION}

A giant a part of machine learning involves dataset pre processing. The information archives from every of those sources come back otherwise formatted, and contain components that we tend to don't actually need (the photos section of our FB information for example). the Hangouts information is formatted to a small degree otherwise from the Face book information, and therefore the LinkedIn messages square measure in an exceedingly CSV format. Our goal, with of these datasets, is to only produce one unified file that contains pairs within the variety of (FRIENDS_MESSAGE, YOUR_RESPONSE). This script can produce 2 completely different files. One is going to be a Numpy object (conversationDictionary.npy) that contains all of the input output pairs. the opposite are going to be an 
outsized txt file \&\#40; conversationData.txt\&\#41; that contains these pairs in sentence type, one when the opposite.

\subsection{Chatbot Text Classification Approaches $>$ PATTERN MATCHERS \\ $>$ ALGORITHMS \\ $>$ NEURAL NETWORKS}

Regardless of which kind of classifier is employed, the end-result could be a response. Sort of a musical instrument, there will be further "movements" related to the machinery. A response will build use of external info (like weather, a sports score, an internet search, etc.) however this isn't specific to chatbots, it's simply further code. A response might reference specific "parts of speech" within the sentence, for example: a correct noun. Additionally, the answer (for AN intent) will use provisional logic to harvest totally different replies betting on the "state" of the oral announcement, this may be a chance choice (to insert some 'natural' feeling).

\subsubsection{PATTERN MATCHERS}

Early chatbots used pattern matching to classify text and turn out a response. Usually this can be $\}$ often brought up as "brute force" because the author of the system must describe each pattern that there's a response. A standard arrangement for these patterns is "AIML" (artificial intelligence mark up language). Its use of the term "artificial intelligence" is kind of AN exaggeration, but that's another story.

A simple pattern matching definition:

$<$ aimlversion $=$ "1.0.1" encoding $=$ "UTF-8"?>

$<$ category $>$

$<$ pattern $>$ WHO IS ALBERT EINSTEIN $<$ ppattern $>$

$<$ template $>$ Albert Einstein was a German

physicist. $</$ template $>$

$</$ category $>$

$<$ category $>$

$<$ pattern $>$ WHO IS Isaac NEWTON $<$ pattern $>$

$<$ template $>$ Isaac Newton was a English physicist and mathematician. $</$ template $>$

$</$ category $>$

$<$ category $>$

$<$ pattern $>$ DO YOU KNOW WHO * IS $<$ /pattern $>$

$<$ template $>$

$<$ srai $>$ WHO IS $<$ star $></$ srai $>$

$</$ template $>$
$</$ category $>$

$</$ aiml $>$

The machine then produces:

Human: Do you know who Albert Einstein is Robot: Albert Einstein was a German physicist?

\subsubsection{ALGORITHMS}

Multinomial Naive Bayes is that the classic rule for text classification and information processing. For associate degree instance, let's assume a group of sentences square measure given that square me a sure happiness to a selected category. With new input sentence, every word is counted for its incidence and is accounted for its commonality and every category is assigned a score. The very best scored category is that the possibly to be related to the input sentence.

For example Sample Training set

class: greeting

"How you doing?"

"good morning"

"hi there"

Few sample Input sentence classification:

input: "Hello good morning"

term: "hello" (no matches)

Term: "good" (class: greeting)

term: "morning" (class: greeting)

classification: greeting $($ score $=2)$

With the assistance of equation, word matches are found for given some sample

sentences for every category. Classification score identifies the category with the very best term matches however it additionally has some limitations. The score signifies that intent is possibly to the sentence however doesn't guarantee it's the right match. Highest score solely provides the theory of relativity base.

\subsubsection{NEURAL NETWORKS}

Artificial neural networks, made-up within the 1940's, are the simplest way of calculative Associate in Nursing output from Associate in Nursing input (a classification) exploitation weighted connections ("synapses") that are calculated from continual iterations through coaching information. every experience the coaching information alters the weights such the neural network produces the output with larger "accuracy" (lower error rate).A neural network structure: nodes (circles) and synapses (lines). There's 
not a lot of new concerning these structures, except today's software system is exploitation a lot of quicker processors and may work with lots additional memory. the mixture of remembering and speed is crucial once you're doing many thousands of matrix multiplications (the neural network's essential math's operation). As within the previous methodology, every category is given with some variety of example sentences. All over again every sentence is attenuated by word (stemmed) and every word becomes Associate in nursing input for the neural network. The conjunction weights are then calculated by iterating through the coaching information thousands of times, on every occasion adjusting the weights slightly to larger accuracy. By recalculating back across multiple layers ("back-propagation") the weights of all synapses are graduated whereas the results are compared to the coaching information output. These weights are $\}$ sort of a 'strength' measure, in a very nerve cell the conjunction weight is what causes one thing to be additional unforgettable than not. You keep in mind an issue additional as a result of you've seen it additional times: on every occasion the 'weight' will increase slightly. At some purpose the adjustment reaches some extent of decreasing returns, this can be referred to as "over-fitting" and going on the far side this can be counter-productive. The trained neural network is a smaller amount code than Associate in nursing comparable rule however it needs a doubtless giant matrix of "weights". In a very comparatively little sample, wherever the coaching sentences have one hundred fifty distinctive words and thirty categories magnificently delicate, relax, it boils all the way down to matrix operation and a formula for reducing values between -1 and one or another bottom vary. even as there square measure variations in pattern matching code and in algorithms, there square measure variations in neural networks, some further advanced than others. the basic machinery is that identical. The essential work is that of classification. The mechanical instrument is tuned in to nothing regarding music theory, like wise chatbot machinery is tuned in to nothing regarding language. Chatbot machinery is looking for patterns in collections of terms; each term is reduced to a token. Throughout this machine words haven't got any which means apart from his or her checkered existence at intervals coaching job info. [38]

In this project under firstly improve performance and accuracy through neural Network. Neural take a different Approach to problem solving then that of conversational Agent. Conversational Agent use an algorithmic approach. i.e. the agents follows a set of instructions in order to solve a problem, neural network process information's in a similar way the human brain does. The chatbot are first trained with the actual data. Training a chatbot happens at much faster and larger scales then you teach a human.

Chat bots use pattern matching to classify the text and manufacture an acceptable response for the user. a typical structure of those is "Artificial intelligence Markup language" (AIML).

A simple pattern matching definition:

$<$ aiml version $=" 1.0 .1 "$

Encoding = "UTF-8"?>

$<$ category $>$

$<$ pattern $>$ What Is Your Name? $<$ pattern $>$

$<$ template $>$ My Name Is Namah.. $<$ /template $>$

$</$ category $>$

The Chatbot then Gives and output:

User: What is Your Name?

Chatbot: My name Is Namah.

\subsubsection{PATTERN MATCHING ALGORITHM}

AIML interpreter tries to match word by word to achieve longest pattern matching and take a look at to search out that the most effective one is. This behavior may be represented with Graph master set of files and a directory containing a group of nodes that is named node master and branches represents initial words of all patterns and wildcard symbols.

\subsection{CHATBOT BENEFITS}

\section{NLP SUPER CHARGED :}

chatbots supply nice promise for client savings, and even larger blessings once it involves prices. Here could be a exposure of different benefits:

\section{> IMMEDIATE ASSISTANCE:}

Chatbots never sleep and never put you on hold! They are available 24 hours a day, seven days a week for real time interaction.

$>$ MORE EFFICIENT SERVICE:

Chatbots can find the precise answer customers need in any connected knowledge base.

\section{HUMAN-LIKE ENGAGEMENT:}

Like a human assistant, the chatbot offers a personalized, one-to-one experience, in a conversational style.

COST AND TIME SAVINGS: 
International Journal of Trend in Scientific Research and Development (IJTSRD) ISSN: 2456-6470

Chabot's will handle several queries while not human additional complicated activities.

intervention, and permits operators to specialize in

\subsection{COMPARISON TABLE}

The comparison of various techniques that can be used to chatbot is listed as under:-

TABLE OF COMPARISION

\begin{tabular}{|c|c|c|c|c|c|c|c|}
\hline Paper & $\begin{array}{l}\text { Development framework/ } \\
\text { platform/ language used }\end{array}$ & $\begin{array}{l}\text { Usage } \\
\text { of } \\
\text { AIML } \\
\end{array}$ & $\begin{array}{l}\text { Usage } \\
\text { of SQL }\end{array}$ & $\begin{array}{l}\text { Data Corpus } \\
\text { used }\end{array}$ & $\begin{array}{l}\text { Algorithm for } \\
\text { matching } \\
\text { keywords } \\
\end{array}$ & $\begin{array}{l}\text { VUI } \\
\text { usage }\end{array}$ & $\begin{array}{l}\text { VUI } \\
\text { usage }\end{array}$ \\
\hline$[20]$ & AIML interpreter & YES & $\mathrm{NO}$ & A.L.I.C.E. & AIML & NO & YES \\
\hline$[23]$ & AIML interpreter & YES & YES & Wikipedia & $\begin{array}{l}\text { Bayesian } \\
\text { Networks }\end{array}$ & NO & YES \\
\hline$[24]$ & AIML interpreter & YES & YES & VPbot & QA matching & NO & YES \\
\hline$[25]$ & RebeccaAIML & YES & YES & DHIS & AIML & $\mathrm{NO}$ & YES \\
\hline$[26]$ & AIML interprete & YES & $\mathrm{NO}$ & $\begin{array}{l}\text { A.L.I.C.E. }+ \\
\text { Custom corpus }\end{array}$ & AIML & $\mathrm{NO}$ & YES \\
\hline [27] & $\begin{array}{c}\text { Program D; Grails 2.0; } \\
\text { Groovy 1.8.6; MySQL; Java }\end{array}$ & YES & YES & $\begin{array}{l}\text { A.L.I.C.E. }+ \\
\text { EMERGO } \\
\end{array}$ & AIML & NO & YES \\
\hline$[28]$ & $\mathrm{C}++; \mathrm{AIML}$ interpreter & YES & YES & A.L.I.C.E. & AIML & NO & YES \\
\hline [29] & Google ASR; MultiSense & NO & YES & $\begin{array}{l}\text { CNN interview } \\
\text { transcripts }\end{array}$ & $\begin{array}{l}\text { Key term } \\
\text { matching }\end{array}$ & YES & YES \\
\hline$[30]$ & Java; AIML interpreter & $\begin{array}{l}\text { Iflter } \\
\text { YES }\end{array}$ & $\mathrm{NO}_{\text {in }}$ & $\begin{array}{l}\text { BNC; Qur'an; } \\
\text { web FAQs; } \\
\text { A.L.I.C.E. }\end{array}$ & AIML & NO & YES \\
\hline$[31]$ & S-AIML & YES & $\mathrm{eNO}$ & Ch S-AIML & AIML & $\mathrm{NO}$ & YES \\
\hline$[32]$ & Verbot & YES & eNOO & $\begin{array}{c}\text { Verbot } \\
\text { Knowledge base }\end{array}$ & AIML & YES & YES \\
\hline$[33]$ & AIML interpreter & YES & $\mathrm{NO}$ & Custom corpus & AIML & $\mathrm{NO}$ & YES \\
\hline
\end{tabular}

Table 1.1 Comparison table of various techniques which are used chatbot

\subsection{RESEARCH WORK WITH DATASET METHODOLOGY}

Humans square measure forever fascinated with creating their daily lives easier with the implementation of recent technology. "Chatbots" square measure one such means that of technology that helps humans during a heap of how, by serving to them increase sales while providing nice client satisfaction and retention. As chatbots may be applied to any industry, humans World Health Organization wish to try to to irrespective of what's going to be creating use of chatbots to form it easier.

Implementing such technology that makes day to day activities easier involves a good deal of advanced cryptography and algorithms to careful perceive the intent of the user in his/her chats and deliver responses consequently. The mix of immediate response and constant property makes them attractive thanks to extend or replace the net applications trend. However do these machine-controlled programs work? Let's have a glance.

\section{HOW IS THE CHATBOT TRAINED?}

Training a chatbot happens at abundant quicker and bigger scale than you teach a personality's. Human's client Service Representatives square measure given manuals and have them browse it and perceive. Whereas the client Support Chatbot is fed with thousands of spoken communication logs and from those logs, the chatbot is ready to grasp what variety of question needs what variety of answers.

\section{HOW CHATBOTS ACTUALLY WORK?}

The chatbots work by adopting three classification methods:

\subsubsection{PATTERN MATCHERS:}


Bots use pattern matching to classify the text and turn out an acceptable response for the purchasers. a typical structure of those patterns is "Artificial Intelligence Mark up Language” (AIML).

A simple pattern matching example:

A simple pattern matching definition:

$<$ aiml version $=" 1.0 .1 "$

Encoding = "UTF-8"?>

$<$ category $>$

$<$ pattern $>$ Do you know who is $*$ ? $</$ pattern $>$

$<$ template $>$ Who IS.. $</$ template $>$

$</$ category $>$

The machine then gives and output:

Human: Do you know who Abraham Lincoln is?

Robot: Abraham Lincoln was the US President during American civil war.

Chatbot is aware of the solution solely as a result of his or her name is within the associated pattern. Similarly, chatbots reply to something relating it to the associated patterns. however it can't transcend the associated pattern. to require it to a sophisticated level algorithms will facilitate.

\subsubsection{Artificial Neural Networks}

Neural Networks unit the only manner of scheming the output from the input practice weighted connections that unit calculated from perennial iterations whereas working the knowledge.

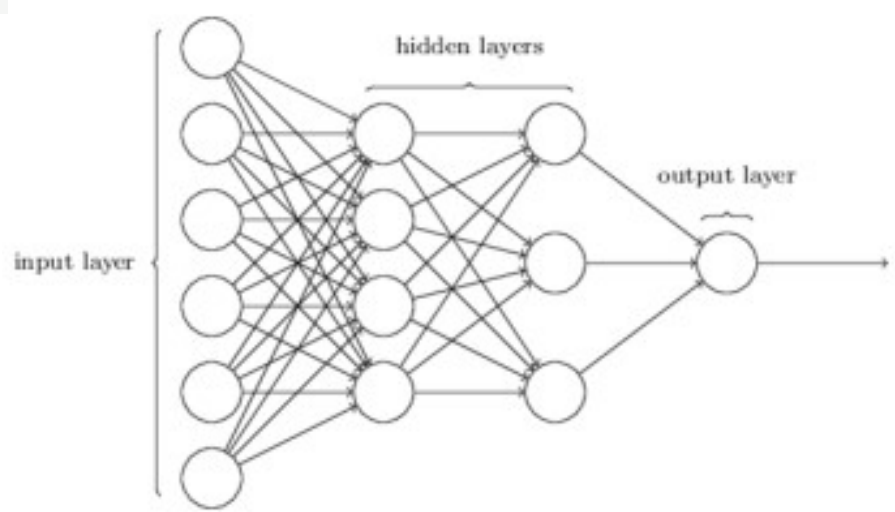

Figure 3.3 multi-layer artificial neural networks [37]
As mentioned earlier here, in addition, each sentence is diminished into all completely different words and each word then is utilized as input for the neural networks. The weighted connections unit of measurement then calculated by all completely different

Entities: Entity primarily represents a thought in your Chatbot. it'd be a payment system in your Ecommerce Chatbot.

$>$ Intents: it's primarily the action chatbot ought to perform once the user say one thing. as an example, intent will trigger same issue if user sorts "I need to order a red try of shoes", "Do you've got red shoes? I need to order them" or "Show ME some red try of shoes", all of those user's text show trigger single command giving users choices for Red try of shoes.

Context: once a NLU algorithmic program analyzes a sentence, it doesn't have the history of the user speech. It implies that if it receives the solution to a matter it's simply asked, it'll not keep in mind the question. For differentiating the phases throughout the chat speech, it's state ought to be hold on. It will either be flags like "Ordering Pizza" or parameters like "Restaurant: 'Dominos"'. With context, you'll simply relate intents with no got to understand what the gesture was. [39]

\subsubsection{NLP (Natural Language Processing)}

Natural Language process (NLP) Chatbot takes some combination of steps to convert the customer's text or speech into structured information that's accustomed choose the connected answer. a number of the language process steps are:

Sentiment Analysis: Tries to be told if the user has an honest expertise or if the once some purpose the chat ought to be forwarded to the human.

Tokenization: The human language technology divides a string of words into items or tokens that area unit lingually symbolic or area unit otherwise helpful for the applying.

$>$ Named Entity Recognition: The chatbot program model appearance for classes of words, just like the name of the merchandise, the user's name or address, whichever information is needed.

Normalization: The Chatbot program model processes the text in a trial to seek out common orthography mistakes or craft errors which may the 
user intent to convey. this offers additional human like impact of the Chatbot to the users.

$>$ Dependency Parsing: The Chatbot appearance for the objects and subjects- verbs, nouns and customary phrases within the user's text to seek out dependent and connected phrases those users could be attempting to convey.

Like most of the Applications, the Chatbot is additionally connected to the information. The cognitive content or the information of knowledge is employed to feed the chatbot with the data required to relinquish an appropriate response to the user. Information of user's activities and whether or not or not your chatbot was able to match their queries, is captured within the information store. human language technology interprets human language into data with a mix of patterns and text which will be mapped within the real time to seek out applicable responses. There are a unit human language technology services and applications programming interfaces that area unit accustomed build the chatbots and create it potential for all variety of businesses, small. Medium and enormous scale. The most purpose here is that good Bots have the potential to assist increase your client base by up the client support services and as a result boosts the sales furthermore as profits. in an exceedingly few years, you'll even see chatbots doing business negotiations higher humans.

\subsection{DIFFERENT CLASSIFIERS}

Machine Learning, Neural Networks and Algorithms

An introduction to a number of the principles behind chatbots. This article is an element of a series regarding Chatbots and Machine Learning. Previous articles that were printed during this series may be found here:

Following the previous article regarding "The Core of the trendy Chatbot," this text provides a deeper understanding regarding the technologies required for chatbots.

\subsubsection{MACHINE LEARNING}

NLP (Natural language processing) and Machine Learning ar each fields in engineering involving AI (Artificial Intelligence). Machine learning may be applied in many alternative fields. human language technology takes care of "understanding" the tongue of the human that the program (e.g. chatbot) is attempting to speak with. This understanding allows the program e.g. chatbot) to each interpret input and turn out output within the kind of human language. The machine "learns" and uses its algorithms through supervised and unsupervised learning. Supervised learning means that to coach the machine to translate the input file into a desired output worth. In different words, it assigns associate inferred operate to information |the info| the information $\}$ in order that newer samples of data can provide an equivalent output for that "learned" interpretation. unsupervised learning means that discovering new patterns within the knowledge with none previous data and coaching. The machine itself assigns associate inferred operate to the info through careful analysis and extrapolation of patterns from information. The layers are for associate alyzing the info in an hierarchal method. This can be to extract, with hidden layers, the feature through supervised or unsupervised learning. Hidden layers are a part of the info process layers in an exceedingly neural network. Featured CBM : Building associate IBM Watson powered AI Chatbot.

\subsubsection{NEURAL NETWORKS}

Neural networks are one among the training algorithms used among machine learning. They contains completely different layers for analyzing and learning knowledge. each hidden layer tries to notice patterns on the image. once a pattern is detected subsequent hidden layer is activated and then on. the image of the Audi A7 higher than illustrates this utterly. the primary layer detects edges. Then the subsequent layers mix different edges found within the knowledge, ultimately a specific layer tries to notice a wheel pattern or a window pattern. betting on the number of layers, it'll be or not be ready to outline what's on the image, during this case a automotive. The additional layers in an exceedingly neural network, the additional is learned and also the additional correct the pattern detection is. Neural Networks learn and attribute weights to the connections between the various neurons anytime the network processes knowledge. this implies subsequent time it comes across such an image, it'll have learned that this explicit section of the image is maybe related to as an example a tire or a door. Featured CBM : unsupervised Deep Learning for Vertical colloquial Chatbots. [40]

\subsubsection{MACHINE LEARNING ALGORITHMS}

This chapter shows a number of the foremost vital machine learning algorithms, additional data 
regarding algorithms may be found via the subsequent links. [1][2][3]

\section{DECISION TREE ALGORITHMS}

In this rule a call tree is employed to map choices and their potential consequences, as well as possibilities, prices and utilities. This methodology permits the matter to be approached logically and stepwise to urge to the correct conclusion. a very important rule that evolved from this rule is that the Random Tree rule. This rule uses multiple trees to avoid over fitting that always happens with victimisation call trees.

\section{> BAYESIAN ALGORITHMS}

Applies Bayesian theorem for regression And classification issues involved likelihood. It tries to point out the probabilistic relationship between completely different variables and verify, given the variables, that class it additional probably belongs to.

\section{$>$ REGRESSION ALGORITHMS}

Well suited to applied math machine learning, regressions ask for to model the link between variables. By perceptive these relationships you aim to ascertain a operate that additional or less mimics this relationship. This mean that once you observe additional variables you'll be able to say with some confidence and with a margin of error, wherever they'll lay on the operate.

\section{$>$ SUPPORT VECTOR}

The support vector rule is employed within the grouping of points on a dimensional plane. The grouping is completed by making a hyper plane that separates the teams with a margin that's as wide as

potential. This helps with the classification and is employed as an example in advertising or human RNA junction.

\section{ENSEMBLE METHODS}

Ensemble strategies mix varied weaker supervised learning algorithms. a mixture of terribly completely different models can sometimes turn out higher results. By combining the varied strategies you'll be able to handle bias with bound models, cut back the variance and cut back overfitting by averaging it out additional.

\section{$>$ CLUSTERING ALGORITHMS}

The main purpose of this rule is to cluster the offered knowledge into teams, wherever the info points in such a bunch ar additional almost like each different one another than those in other teams. the additional vital cluster strategies ar hierarchal, centroid, distribution and density.

ASSOCIATION RULE LEARNING ALGORITHMS

This is regarding the foundations that may be established between the itemsets and also the transactions for this stuff and item sets. The relation between $\mathrm{X}$ and $\mathrm{Y}$, therefore the likelihood of once you acquire $\mathrm{X}$ you furthermore mght acquire $\mathrm{Y}$. This rule is found within the info by perceptive the thingsets and also the items in this.

\section{ARTIFICIAL NEURAL NETWORK ALGORITHMS}

Artificial Neural Network algorithms ar galvanized by the human brain.the bogus neurons ar interconnected and communicate with one another. every affiliation is weighted by previous learning events and with every new input of information additional learning takes place. heaps of various algorithms ar related to Artificial Neural Networks and one among the foremost vital is Deep learning. Associate example of Deep Learning may be seen within the image higher than. it's particularly involved with building a lot of larger complicated neural networks.

\section{$>$ DIMENSIONALITY ANALYSIS ALGORITHMS}

Dimensionality is regarding the number of variables within the information and therefore the dimensions they belong to. this sort of study is aimed toward reducing the number of dimensions with the associated variables whereas at a similar time holding

a similar data. In alternative words it seeks to get rid of the less pregnant information whereas at a similar time making certain a similar final result.

\subsubsection{CHATBOTS WITH MACHINE LEARNING: BUILDING NEURAL CONVERSATIONAL AGENTS}

Using machine learning approaches to make good chatbots have you ever talked to Siri, Alexa, or Cortana to line up Associate in Nursing alarm, decision friends, or organize a meeting? many folks might agree that despite their quality in common and routine tasks, it's tough to force colloquial agents to speak on general, typically philosophical topics. The Statsbot team invited a knowledge mortal, Dmitry Persiyanov, to clarify a way to fix this issue with 
neural colloquial models and build chatbots mistreatment machine learning. Interacting with the machine via language is one among the necessities for general computing. This field of AI is named dialogue systems, spoken dialogue systems, or chatbots. The machine must give you with Associate in Nursing informative answer, maintain the context of the dialogue, and be indistinguishable from the human (ideally). In follow, the last demand isn't approachable however, however fortuitously, humans ar able to speak with robots if they're useful, typically funny, and fascinating interlocutors. There are 2 major sorts of dialogue systems: goal-oriented (Siri, Alexa, Cortana, etc.) and general speech (Microsoft Tay bot). The former facilitate folks to unravel everyday issues mistreatment language, whereas the latter decide to speak with folks on a good vary of topics. In this post, $\mathrm{i}$ will be able to provide you with a comparative summary of general speech dialogue systems supported deep neural networks. i will be able to describe main design varieties and ways in which to advance them. Also, there'll be a great deal of links to papers, tutorials, and implementations. I hope this post can eventually become the entry purpose for everybody World Health Organization needs to make chatbots with machine learning. If you scan this post until the tip, you may be able to train your own colloquial model. Ready?

Go ahead :) I'm about to discuss with repeated neural networks and word embeddings, therefore you must shrewdness they add order to simply follow the article. For people who got to refresh their information, I've ready nice tutorials at the tip of the article for you.

\subsubsection{NOTE ON DIALOGUE DATA REPRESENTATION}

Before going deeper, we should always discuss what dialogue datasets appear as if. All models delineate below ar trained on pairs (context, reply). Context is many sentences (maybe one) that preceded the reply. The sentence is simply a sequence of tokens from its vocabulary.

For higher understanding, look into the table. There's a batch of 3 samples extracted from raw dialogue between 2 persons:

$>\mathrm{Hi}$ !

$>$ Hi there.

$>$ How old are you?

$>$ Twenty-two. And you?
$>$ Me too! Wow!

Note the "<eos $>$ " (end-of-sequence) token at the end of each sentence in the batch. This special token helps neural networks to understand sentence bounds and update its internal state wisely.

Some models may use additional meta information from data, such as speaker id, gender, emotion, etc.

Now, we are ready to move on to discussing generative models.

\subsubsection{GENERATIVE MODELS}

We begin with the only colloquial model, supported the paper "A Neural colloquial Model." For modeling dialogue, this paper deployed a sequence-to-sequence (seq2seq) framework that emerged within the neural artificial intelligence field and was with success tailored to dialogue issues. The design consists of 2 RNNs with totally different sets of parameters. The left one (corresponding to A-B-C tokens) is termed the encoder, whereas the correct one (corresponding to $-\mathrm{W}-\mathrm{X}-\mathrm{Y}-\mathrm{Z}$ tokens) is termed the decoder.

\section{HOW DOES THE ENCODER WORK?}

The encoder RNN conceives a sequence of context tokens one at a time and updates its hidden state. once process the entire context sequence, it produces a final hidden state, which contains the sense of context and is employed for generating the solution.

\section{HOW DOES THE DECODER WORK?}

The goal of the decoder is to require context illustration from the encoder and generate a solution. For this purpose, a softmax layer over vocabulary is

maintained within the decoder RNN. At every time step, this layer takes The decoder hidden state and outputs a likelihood distribution over all words in its vocabulary.

Here is however reply generation works:

1. Initialize decoder hidden state with final encoder hidden state ( $\mathrm{h} \_0$ ).

2. Pass token as 1 st input to the decoder and update hidden state (h_1)

3. Sample (or take one with liquid ecstasy probability) 1st word (w_1) from softmax layer (using h_1).

4. Pass this word as input, update hidden state (h_1 $>$ h_2) and generate new word (w_2). 
5. Repeat step four till token is generated or most answer length is exceeded.

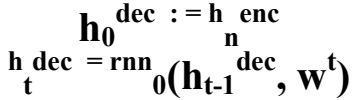

$$
\begin{aligned}
& \mathbf{w}_{t+1} \sim \mathbf{p}_{\mathbf{t}+1}=\operatorname{softmax}\left(\mathrm{g}_{\text {pie }}\left(\mathbf{h}_{t}{ }^{\mathrm{dec}}\right)\right)
\end{aligned}
$$

Reply generation in decoder, for people who prefers formulas rather than words. Here, $w_{-} t$ is that the sampled word on time step $t$; alphabetic character square measure decoder parameters, letter square measure dense layers parameters, g represents dense layers, p-hat could be a likelihood distribution over vocabulary at time step t. Using argmax whereas generating a reply, one can continuously get a similar answer once utilizing a similar context (argmax is settled, whereas sampling is stochastic). The process I've delineated higher than is barely the model logical thinking half, however there's conjointly the model coaching half, that works in a very slightly totally different way - at every cryptography step, we have a tendency to use the proper word nonetheless rather than the generated one ( $\left.w_{-} t\right)$ because the input. In alternative words, at coaching time, the decoder consumes an accurate reply sequence, however with the last token removed and therefore the token prepended. Illustration of decoder logical thinking part. Output at previous time step is fed as input at current time step. The goal is to maximize likelihood of an accurate next word on every time step. a lot of merely, we have a tendency to raise the network to predict consequent word within the sequence by providing it with an accurate prefix. coaching is performed via most chance coaching, that results in classical cross-entropy loss:

$$
L=\sum_{t=1}^{m} \sum_{i=1}^{|V|} I\left[y_{t}=i\right] \log \hat{p}_{t, i}=\sum_{t=1}^{m} \log \hat{p}_{t, y_{t}}
$$

Here, $y_{-} t$ is a correct word in reply at time step $t$.

\subsubsection{MODIFICATIONS OF GENERATIVE MODELS}

Now we've got a basic understanding of sequence-tosequence framework. However can we add a lot of generalization power to such models? There square measure a bunch of ways:

1. Add a lot of layers to encoder or/and decoder RNNs.

2. Use a two-way encoder. There's no thanks to build the decoder two-way thanks to its forward generation structure.

3. Experiment with embeddings. you'll pre-initialize word embeddings or learn them from scratch beside the model.
4. Use a lot of advanced reply generation procedure - beam search. the concept is to not generate a reply "greedily" (by taking arg max for consequent word) however think about the likelihood of longer chains of words and select among them.

5. Build your encoder or/and decoder be convolution. Convents would possibly work abundant quicker than RNNs as a result of they'll be parallelized with efficiency.

6. Use associate attention mechanism. Attention was at the start introduced in neural artificial intelligence papers, and has become a really widespread and powerful technique.

7. Pass the ultimate encoder state at every time step to the decoder. The decoder sees the ultimate encoder state just once and so could forget it. an honest plan is to pass it to the decoder in conjunction with word embedding.

8. totally different encoder/decoder state sizes. The model I delineated higher than needs the encoder and decoder to own a similar hidden state size (because we have a tendency to initialize the decoder state with the ultimate encoder's state). You'll get obviate this demand by adding a projection (dense) layer from the encoder final state to the initial decoder state.

9. Use characters rather than words or computer memory unit combine coding for building vocabulary. Character-level models square measure value considering as they work quicker attributable to a smaller vocabulary and that they will perceive words that don't seem to be in their vocabulary. Computer memory unit combine coding (BPE) is that the better of each worlds. the concept is to search out the foremost frequent pairs of tokens in a very sequence and merge them into one token. 


\section{RESULT}

4.1 CHATBOT SCREEN SHOT:

In this Screenshot, the data set is being loaded in the cahtbot.

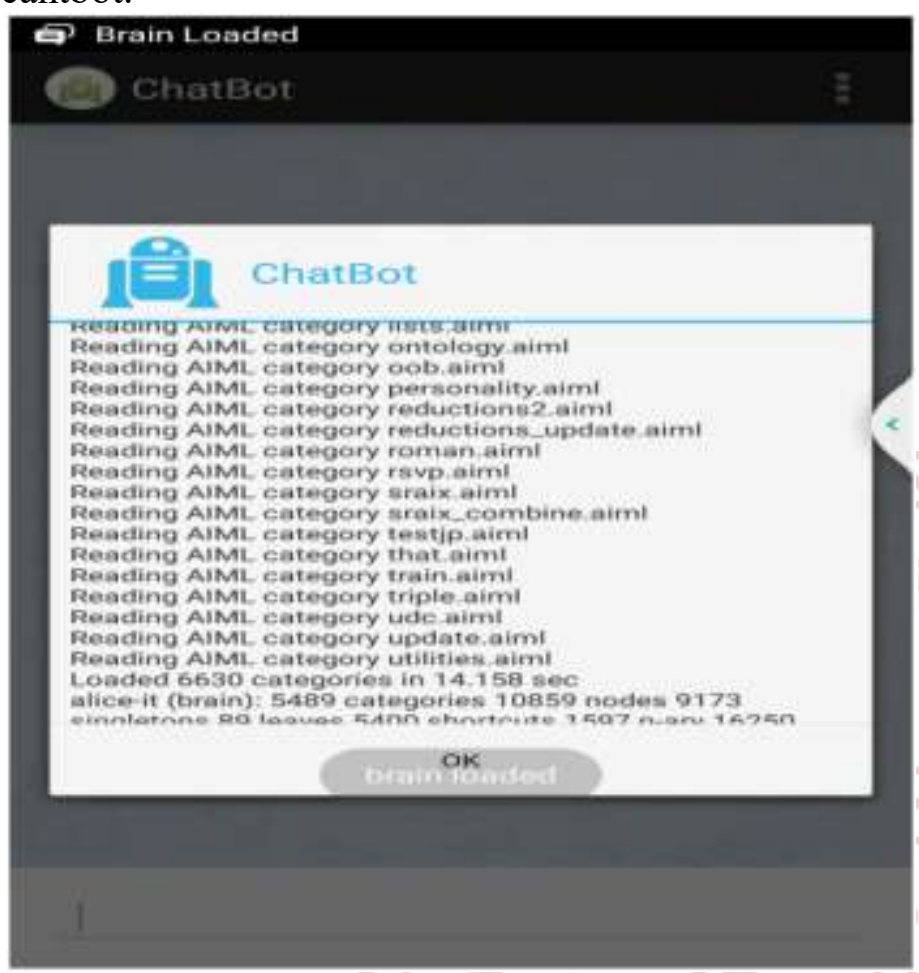

Figure 4.1 Data Set Being Loaded

In this Screenshot, When this blank screen is loaded, then the dataset will open this chatbot blank screen.

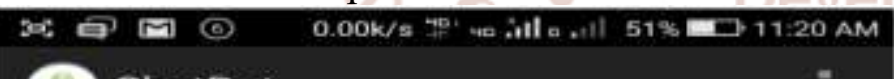

Figure 4.2 Data Set After Loaded
Next the Screenshot show when the user asked for a quary and say hello

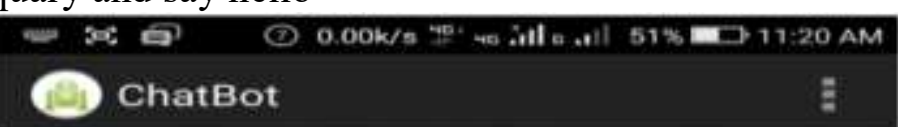

\begin{tabular}{|c|c|c|c|c|c|c|c|c|c|c|c|c|}
\hline $\mathbf{q}^{\prime}$ & $w^{2}$ & & & $r^{*}$ & & s & $y^{6}$ & $\mathbf{u}$ & & $i^{\prime \prime}$ & 0 & $p^{0}$ \\
\hline a & & s & d & . & f & g & 1 & t & $\mathbf{j}$ & k & & 1 \\
\hline$\Delta$ & & z & $x$ & & C & $v$ & t & D & $n$ & $n$ & & $x$ \\
\hline 212: & & $?$ & \multicolumn{7}{|c|}{ English } & & & $\checkmark$ \\
\hline
\end{tabular}

Figure 4.3 User Can Quary

Next the Screenshot show when the user asked for a quary and say helloand then chatbot responded hi it's great to see you.

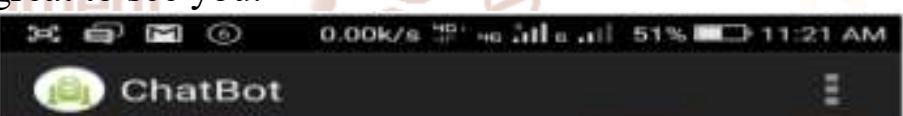

hetso

Hit it's great to see you

Figure 4.4 Chatbot Response User Quary Meessage 
Next the Screenshot show when the user asked for a quary and chatbot responded by user quary.
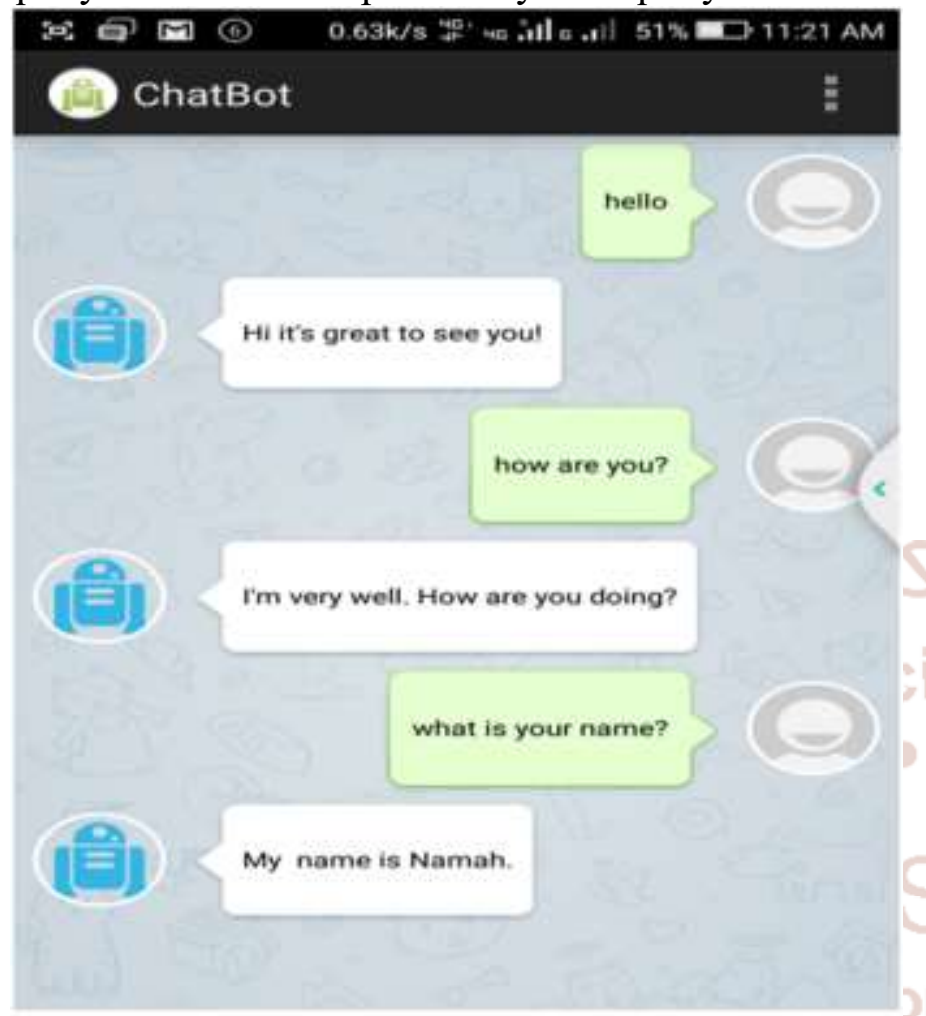

Figure 4.5 Conversation User And Chatbot

\section{CONCLUSION}

In this thesis, we show some integrated systems which are added AIML based chatbot to their system to make interface with user. Besides, user gets unlimited full time chatting service which make interest user to use this particular service. this ChatBot application will give users easy experience and easy feedback. Users can send questions directly to the agent and get an immediate response from the agent. A chatbot is a conversation agent wherever a computer program is designed to emulate an intelligent conversation. It can take user input in many formats like text, voice, emotions etc. For this purpose, many open-source platforms are available. Currently built for a one domain, we can work on this Multiplayer domain. The paper describes the modules and resources required for running the agent on both interfaces, as well as the evaluation results obtained from two assessment studies concerning the interaction design of these two agent interfaces. The feedback gathered from the studies will enable us to improve the applications in terms of service, performance and usability. It will act as a good virtual assistant for perform multi-dimensional analysis.
Automaton based mostly good chat-bot ${ }^{e e}$ could be a terribly promising system and aims to boost the user and client expertise.

\section{FUTURE SCOPE}

Conversational Agent application will only work on English language. Later it can work in several languages and in this application, single domain is used, later it can work on multiple domains. Just now we are using text messages in chatbot. But in future, we can send it to voice message, video chat, attachment share, location share, images share, documents share. All these features can also be added.

\section{REFERENCES}

1. R. Mahapatra, N. Sharma, A. Trivedi, and C. Arnan, "Adding interactive interlace to egovernment systems using aiml based chatterbots," in Software Engineering (CONSEG), 2012 CSI Sixth International Conference on. IEEE, 2012, pp. 1-6.

2. B. A. Shawar and E. Atwell, "Using dialogue corpora to train a chatbot," in Proceedings of the Corpus Linguistics 2003 conference, 2003, pp. 681-690.

3. A. Augello, A. Santangelo, S. Sorce, G. Pilato, A. Gentile, A. Genco, and S. Gaglio, "A multimodal interaction guide for pervasive services access," in Pervasive Services, IEEE International Conference on. IEEE, 2007, pp. 250-256.

4. D. De Orlando and F. Giovanni, "An integrated system, with natural language management, for the monitoring activities in e-Iearning environments," in Complex, Intelligent and Software Intensive Systems, 2008. CISIS 2008.International Conference on. IEEE, 2008, pp. 965970.

5. F. Mikic, J. C. Burguillo, M. Llamas, D. Rodrfguez, E. RodrfguezetaI. "Charlie: An aimlbased chatterbot which works as an interface among Ines and humans," in EAEEIE Annual Conference, 2009. IEEE, 2009, pp. 1-6.

6. S. du Preez, M. Lall, and S. Sinha, "An intelligent web-based voice chat bot," in EUROCON 2009, EUROCON'09.IEEE. IEEE, 2009, pp. 386-391.

7. A. Augello, G. Pilato, A. Machi, and S. Gaglio, "An approach to enhance chatbot semantic power and maintainability: Experiences within the frasi project," in Semantic Computing (ICSC), 2012 
IEEE Sixth International Conference on. IEEE, 2012, pp. 186-193.

8. S. Negi, S. Joshi, A. Chalamalla, and L. V. Subramarnam, "Automatically extracting dialog models from conversation transcripts," in Data Mining, 2009. ICDM'09.Ninth IEEE International Conference on. IEEE, 2009, pp. 890-895.

9. J. T. C. Tan and T. Inamura, "Extending chatterbot system into multimodal interaction framework with embodied contextual understanding," in Proceedings of the seventh annual ACMI lEEE international conference on Human-Robot Interaction. ACM, 20।2, pp. 251-252.

10. A. Augello, G. Saccone, S. Gaglio, and G. Pilat, "Humorist bot: Bringing computational hum our in a chat-bot system," in Complex, Intelligent and Software Intensive Systems, 2008. CISIS 2008.International Conference on. IEEE, 2008, pp. 703-708.

11. M. D. Leonhardt, L. Tarouco, R. M. Vicari, E. R. Santos, and M. D. S. d. Silva, "Using chatbots for network management training through problembased oriented education," in Advanced Learning Technologies, 2007. ICALT 2007.Seventh IEEE International Conference on. IEEE, 2007, pp. 845-847.

12. G. Pilato, A. Augello, G. Vassallo, and S. Gaglio, "Sub-symbolic semantic layer in eyc for intuitive chat-bots," in Semantic Computing, 2007. ICSC 2007.International Conference on. IEEE, 2007, pp. 121-128.

13. G. Pilato, A. Augello, and S. Gaglio, "A modular architecture for adaptive chatbots," in Semantic Computing (lCSC), 201 I Fifth IEEE International Conference on. IEEE, 2011, pp. 177-180.

14. Turing, A. M. (1950). Computing machinery and intelligence. Mind, 59(236), (pp. 433-460).

15. Weizenbaum, J. (1966). ELIZA - a computer program for the study of natural language communication between man and machine. Communications of the ACM, 9(1), (pp. 36-45).

16. Colby, K. M. (1981). Modeling a paranoid mind. Behavioral and Brain Sciences, 4(4), (pp. 515534).

17. Wallace, R. S. (2003). The elements of AIML style. Alice AI Foundation.
18. Shawar, B. A., \& Atwell, E. (2004). A Chatbot as a novel corpus visualization tool.In $L R E C$.

19. Galvão, A. M., Barros, F. A., Neves, A. M., \&Ramalho, G. L. (2004, July). Persona-aiml: An architecture developing Chatterbots with personality. In Proceedings of the Third International Joint Conference on Autonomous Agents and Multiagent Systems, Vol. 3 (pp. 12661267). IEEE Computer Society.

20. Sansonnet, J. P., Leray, D., \& Martin, J. C. (2006). Architecture of a framework for generic assisting conversational agents.In Intelligent Virtual Agents (pp. 145-156).Springer Berlin/Heidelberg.

21. Wallace, R. S. (2007). Chapter 00.The anatomy of ALICE. Alice AI Foundation.

22. Datta, A. (2008). Contextual flow in Chatbot conversations. Retrieved from https://cse.iitk.ac.in.

23. Lokman, A. S., \& Zain, J. M. (2009, August). An architectural design of Virtual Dietitian (ViDi) for diabetic patients. In Computer Science and Information Technology, 2009.ICCSIT 2009. 2nd IEEE International Conference on (pp. 408-411). IEEE.

24. Kethuneni, S., August, S. E., \& Vales, J. I. (2009, October). Personal health care assistant/companion in virtual world.In 2009 AAAI Fall Symposium Series.

25. Rahman, J. (2012). Implementation of ALICE Chatbot as domain specific knowledge bot for BRAC U (FAQbot). Retrieved from http://dspace.bracu.ac.bd

26. Van Rosmalen, P., Eikelboom, J., Bloemers, E., Van Winzum, K., \& Spronck, P. (2012, October). Towards a game-Chatbot: Extending the interaction in serious games. In European Conference on Games Based Learning (p. 525). Academic Conferences International Limited.

27. Khanna, A., Pandey, B., Vashishta, K., Kalia, K., Pradeep Kumar, B., \& Das, T. (2015). A study of today's AI through Chatbots and rediscovery of machine intelligence. International Journal of $u$ and $e$-Service, Science and Technology, 8, (pp. 277-284).

28. Yu, Z., Pap Angelis, A., \& Rudnicky, A. (2015, March). Tick Tock: A non-goal-oriented multimodal dialog system with engagement 
awareness. In Proceedings of the AAAI Spring Symposium.

29. Abu Shawar, B., \& Atwell, E. (2016). Automatic extraction of Chatbot training data from natural dialogue corpora. In RE-WOCHAT: Workshop on Collecting and Generating Resources for Chatbots and Conversational Agents-Development and Evaluation Workshop Programmed (May 28 $8^{\text {th }}$, 2016) (p. 29).

30. Augello, A., Gentile, M., Weideveld, L., \&Dignum, F. (2016). A model of a social Chatbot. In Intelligent Interactive Multimedia Systems and Services 2016 (pp. 637-647). Springer International Publishing.

31. Hatwar, N., Patil, A., \& Gondane, D. (2016). AI based Chatbot. International Journal of Emerging Trends in Engineering and Basic Sciences, Vol. 3, Issue 2 (March-April 2016), (pp.85-87)

32. Behera, B. (2016). Chappie- A semi-automatic intelligent Chatbot. Retrieved from https://www.cse.iitb.ac.in.

33. Mhatre, N., Motani, K., Shah, M., \& Mali, S. (2016). Donna interactive Chat-bot acting as a personal assistant. International Journal of Computer Applications, 140(10).

34. McTear, M., Callejas, Z., \&Griol, D. (2016). Creating a conversational interface using Chatbot technology. In The Conversational Interface ( $p p$. 125-159). Springer International Publishing.
35. Doshi, S. V., Pawar, S. B., Shelar, A. G., Kulkarni, S. S. (2017). Artificial Intelligence Chatbot in android system using open source Program-O. International Journal of Advanced Research in Computer and Communication Engineering, Vol. 6, Issue 4 (April 2017), (pp. 816-821).

36. Åberg, J. (2017). Chatbots as a mean to motivate behavior change: How to inspire proenvironmental attitude with Chatbot interfaces (Dissertation).

37. https://chatbotslife.com/how-neural-networkswork-ff4c7ad371f7 (Date last accessed 02-march2018).

38. Rana, A., Kumar, A., Sharma, A., (2016) Neural network radial basis Function classifier for earthquake data using a FOA, International Journal of Advanced Research Vol. 4, Issue 8, (pp. 537-540).

39. Rana, A., Sharma A., (2014) Optimization of radial basis neural network by mean of amended fruit fly optimization algorith, Journal of Computer and mathematical Sciences Vol. 5, Issue 3, (pp. 258-331).

40. Rani, U., Rana, A., Barjtya, S., (2014) Review on Various scheduler used by Hadoop framework, International Journal of Engineering and Computer Science Vol. 6, Issue 9. 